\title{
ELECTRICAL SAFETY IN SPINAL CORD STIMULATION: CURRENT DENSITY ANALYSIS BY COMPUTER MODELING
}

\author{
W.A. Wesselink, J. Holsheimer \\ Institute for Biomedical Technology, University of Twente \\ P.O. box 217, 7500 AE, Enschede, the Netherlands
}

\begin{abstract}
The possibility of tissue damage in spinal cord stimulation was investigated in a computer modeling study. A decrease of the electrode area in monopolar stimulation resulted in an increase of the current density at the electrode surface. When comparing the modeling results with experimental data from literature, it was concluded that even with a small electrode area $\left(0.7 \mathrm{~mm}^{2}\right)$ tissue damage in spinal cord stimulation is improbable.
\end{abstract}

\section{INTRODUCTION}

Tissue damage resulting from electrical stimulation is usually ascribed to the following factors: (1) the induction of irreversible electrochemical reactions at the electrode-tissue interface; (2) processes associated with the current passing through the tissue [1].

In prolonged spinal cord stimulation (SCS) short duration, charge balanced pulses are given by electrodes of inert material, thus minimizing irreversible chemical reactions. Furthermore, the electrodes are in the dorsal epidural space, usually a few millimeters apart from the nervous tissue in the spinal cord and the dorsal roots. Only the dura mater and the epidural fat cells are in the close vicinity of the electrodes.

The threshold of electrically induced tissue damage was determined experimentally by Agnew et al. [1]. They concluded that the charge per phase and the charge density per phase (of a charge balanced, biphasic pulse) at the electrode surface are associated with the tissue damage. These parameters can be derived frorn the current density at the electrode surface, and the total current per phase from the electrode. Results obtained from a modeling study are compared with these experimental data to evaluate the electrical safety of SCS.

\section{METHODS}

Current densities were calculated by a computer model, which consists of two parts [2]. The first part comprises a threedimensional cubical cell structure with variable cell dimensions. Each cell has a specified conductivity, resulting in an inhomogeneous volume conductor model. The compartments represent the gross anatomy of the spinal cord e.g. gray and white matter, epidural space, dura mater, vertebral bone, cerebrospinal fluid, and a layer representing surrounding tissues. A transverse section of the mid-cervical volume conductor model, characterized by a $2.4 \mathrm{~mm}$ spacing between the spinal cord and the epidural contact, is shown in figure 1 .

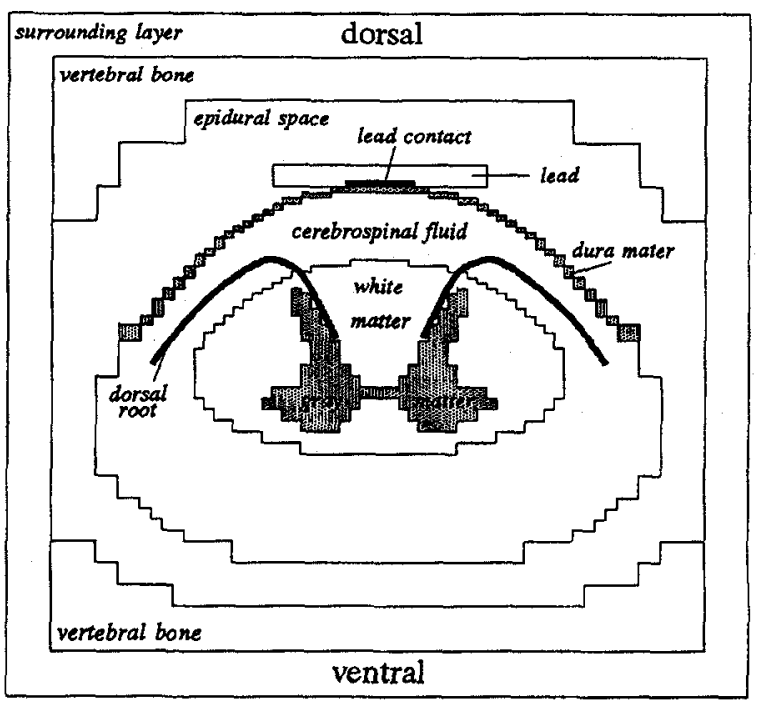

Figure 1: Transverse section of the mid-cervical spinal cord model

The potential field, induced by the epidural lead contacts, is calculated by solving the discretized Laplace equation by a Red-Black Gauss-Seidel iteration. A finite difference method was also used to calculate the current density. Finite differences of second order represent the current density at regular grid points, whereas finite differences of first order are used at electrode and border points.

The second part of the model represents the electrical behavior of the myelinated nerve fibers in the spinal cord, as described by McNeal [3]. A fiber model of a $12 \mu \mathrm{m}$ dorsal column fiber with collaterals, placed at the dorsomedial border of the spinal cord, was used. Threshold voltages for the excitation of this fiber were calculated for a $210 \mu \mathrm{s}$ rectangular stimulus pulse. 
In this study, a mid-cervical spinal cord model was stimulated monopolarly. The cathode was placed dorsomedially in the epidural space, bordered the dura mater, and had a rectangular shape. Four electrode areas were used, i.e. $0.7,3.15,7.0$, and $12.25 \mathrm{~mm}^{2}$. The smallest cell dimension was $0.2 \mathrm{~mm}$.

\section{RESULTS}

Current density at both the electrode surface and the border of the dorsal columns was considered at the threshold voltage of the dorsomedial nerve fiber. This threshold was $1.2 \mathrm{~V}$ for the largest and $4.8 \mathrm{~V}$ for the smallest electrode.

The current density at the electrode surface was highest at its corners. This maximum current density increased with decreasing electrode area. The value for the $0.7 \mathrm{~mm}^{2}$ electrode was approximately 5 times higher than for the $\mathbf{1 2 . 2 5}$ $\mathrm{mm}^{2}$ electrode. The maximum current density at the border of the dorsal columns was highest with the largest electrode, but only differed $20 \%$ from the smallest electrode. Compared to the current density at the electrode, the maximum current density at the dorsal border of the spinal cord was 5 and 30 times smaller for the $12.25 \mathrm{~mm}^{2}$ and $0.7 \mathrm{~mm}^{2}$ electrodes, respectively.

After calculation of the total current from the electrode, the charge and charge density per pulse were determined. Figure 2 shows the charge per pulse and the maximum charge density per pulse both at the electrode surface (1) and at the border of the dorsal columns (2). The data are related to the four electrode areas. Highest charge density per pulse was found with the smallest electrode.

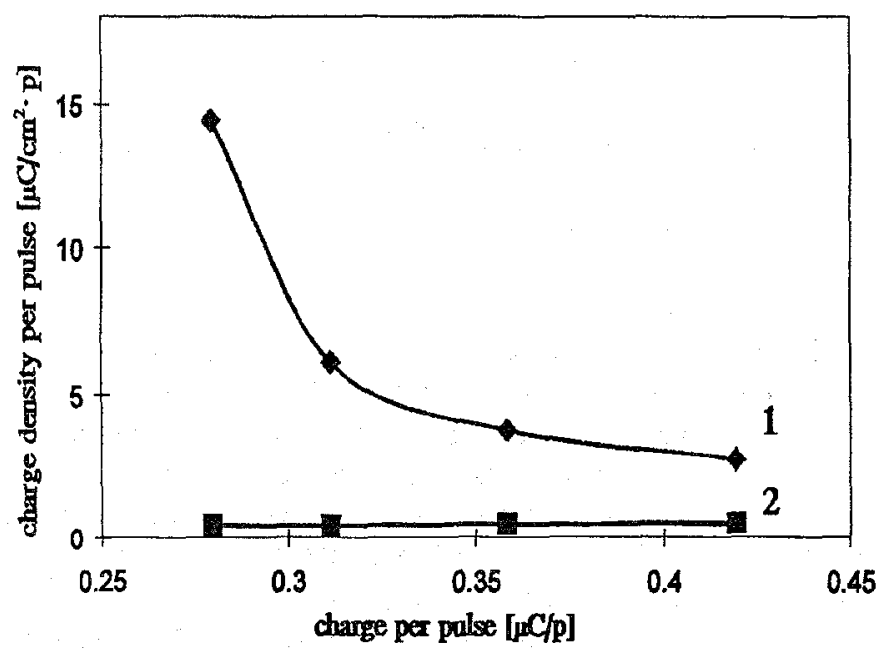

Figure 2: Calculated charge and (max.) charge density per pulse when varying modeled electrode area: 1 . at electrode surface; 2 . at border of spinal cord

As shown in figure 2, the maximum charge density per pulse at the border of the dorsal columns varies slightly $(0.48-0.56$ $\mu \mathrm{C} / \mathrm{cm}^{2} \mathrm{p}$ ) and is 5 to 30 times smaller than the maximum charge density per pulse at the electrode surface.

\section{DISCUSSION}

It was found that a decrease in electrode area resulted in an increase in charge density at the electrode surface needed for excitation of the dorsal column fiber. However, the charge density at the dorsal border of the spinal cord did not change much when the electrode area was varied.

Although the experimentally determined safety limits presented by Agnew et al. [1] were not obtained from spinal cord stimulation, they will be used here for comparison with the computational results. The safety limits proposed by Agnew et al. ranged from a mean charge density of 140 $\mu \mathrm{C} / \mathrm{cm}^{2} \mathrm{ph}$ and a charge of $0.15 \mu \mathrm{C} / \mathrm{ph}$ to a mean charge density of $5 \mu \mathrm{C} / \mathrm{cm}^{2} \mathrm{ph}$ and a charge of $25 \mu \mathrm{C} / \mathrm{ph}$ (charge balanced, biphasic pulses). Taking into account that the maximum stimulus in SCS is usually less than twice the threshold stimulus and that maximum charge densities per pulse were calculated instead of mean values, it can be concluded that even with an electrode area of $0.7 \mathrm{~mm}^{2}$ the charge $(0.56 \mu \mathrm{C})$ and charge density $\left(29 \mu \mathrm{C} / \mathrm{cm}^{2}\right)$ per pulse in monopolar stimulation are still within these limits. This is also true for a $3.6 \mathrm{~mm}$ distance between electrode and spinal cord, resulting in a maximum charge density per pulse of 55 $\mu \mathrm{C} / \mathrm{cm}^{2}$ and a charge per pulse of $1 \mu \mathrm{C}$. Therefore, the model predicts that prolonged electrical stimulation of the spinal cord with this electrode configuration will not result in any tissue damage.

Other parameters that possibly influence the charge and charge density per pulse in spinal cord stimulation, like pulse duration and electrode configuration, will be the subject of research in following modeling studies.

\section{REFERENCES}

W.F. Agnew, D.B. McCreery, T.G.H. Yuen, L.A. Bullara, Effects of prolonged electrical stimulation of the central nervous system, in Neural prostheses: Fundamental studies, Chapter 9, Prentice-Hall, Englewood Cliffs, 1990

J.J. Struijk, J. Holsheimer, G.G. van der Heide, H.B.K. Boom, Recruitment of dorsal column fibers in spinal cord stimulation: Influence of collateral branching, IEEE Trans. on Biomed. Eng., vol.39, pp. 903-912, 1992

D.R. McNeal, Analysis of a model for excitation of myelinated nerve, IEEE Trans. on Biomed. Eng., vol. BME-23, pp. 329-337, 1976 